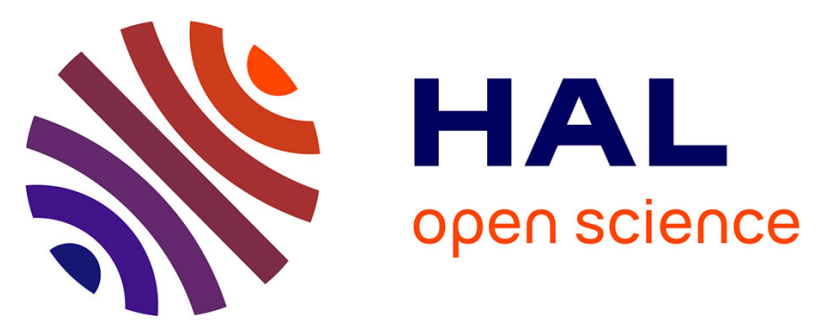

\title{
Investigation of GaN nanowires containing AlN/GaN multiple quantum discs by EBIC and CL techniques
}

Valerio Piazza, Andrey Babichev, Lorenzo Mancini, Martina Morassi, Patrick

Quach, Fabien Bayle, Ludovic Largeau, François Julien, Pierre Râle, Stéphane Collin, et al.

\section{To cite this version:}

Valerio Piazza, Andrey Babichev, Lorenzo Mancini, Martina Morassi, Patrick Quach, et al.. Investigation of GaN nanowires containing AlN/GaN multiple quantum discs by EBIC and CL techniques. Nanotechnology, 2019, 30 (21), pp.214006. 10.1088/1361-6528/ab055e . hal-02182873

\section{HAL Id: hal-02182873 https://hal.science/hal-02182873}

Submitted on 13 Jul 2019

HAL is a multi-disciplinary open access archive for the deposit and dissemination of scientific research documents, whether they are published or not. The documents may come from teaching and research institutions in France or abroad, or from public or private research centers.
L'archive ouverte pluridisciplinaire HAL, est destinée au dépôt et à la diffusion de documents scientifiques de niveau recherche, publiés ou non, émanant des établissements d'enseignement et de recherche français ou étrangers, des laboratoires publics ou privés. 


\title{
Investigation of GaN Nanowires Containing AIN/GaN Multiple Quantum Discs by EBIC and CL techniques
}

Valerio Piazza ${ }^{1 *}$, Andrey V. Babichev ${ }^{2}$, Lorenzo Mancini ${ }^{1}$, Martina Morassi ${ }^{1}$, Patrick Quach ${ }^{1}$, Fabien Bayle $^{1}$, Ludovic Largeau ${ }^{1}$, François H. Julien ${ }^{1}$, Pierre Rale ${ }^{1}$, Stéphane Collin ${ }^{1}$, Jean-Christophe Harmand $^{1}$, Noelle Gogneau ${ }^{1}$, Maria Tchernycheva ${ }^{1}$

${ }^{1}$ Centre de Nanosciences et de Nanotechnologies, Université Paris Sud, Avenue de la Vauve, 91120 Palaiseau, France

${ }^{2}$ ITMO University, 197101 St. Petersburg, Russia

*Corresponding author email: valerio.piazza@u-psud.fr

\begin{abstract}
In this work, nanoscale electrical and optical properties of n-GaN nanowires (NWs) containing GaN/AIN multiple quantum discs (MQDs) grown by molecular beam epitaxy (MBE) are investigated by means of single wire I(V) measurements, electron-beam induced current microscopy (EBIC) and cathodoluminescence (CL) analysis. A strong impact of nonintentional AlN and GaN shells on the electrical resistance of individual NWs is put in evidence. The EBIC mappings reveal the presence of two regions with internal electric fields oriented in opposite directions: one in the MQDs region and the other in the adjacent bottom GaN segment. These fields are found to co-exist under zero bias, while under an external bias either one or the other dominates the current collection. In this way EBIC maps allow to locate the current generation within the wire under different bias conditions and to give the first direct evidence of carrier collection from AIN/GaN MQDs. The NWs have been further investigated by photoluminescence (PL) and cathodoluminescence (CL) analyses at low temperature. CL mappings show that the near band edge emission of GaN from the bottom part of the NW is blue-shifted due to the presence of the radial shell. In addition, it is observed that CL intensity drops in the central part of the NWs. Comparing the CL and EBIC maps, this decrease of the luminescence intensity is attributed to an efficient charge splitting effect due to the electric fields in the MQDs region and in the GaN base.
\end{abstract}

KEYWORDS: GaN, Single Nanowires, Multiple Quantum Discs, EBIC, Cathodoluminescence.

\section{Introduction}

In the past years, semiconductor nanowires (NWs) have raised a huge interest due to their attractive optical properties such as low reflectance, large absorption cross-section and light-trapping phenomena $[1,2]$. In addition, their small foot print allows to relax the mechanical stress arising from the lattice mismatch via lateral surface, strongly decreasing the defect density and thus increasing the material quality compared to their bulk counterparts [3]. These properties are of great interest for the development of III-nitride based devices. Indeed, III-N 2D layers suffer from large dislocation densities when grown on conventional substrates (typically $10^{8}-10^{10} \mathrm{~cm}^{-3}$ ). Such a high defect density strongly affects the optical and electrical properties, thus limiting the efficiency of nitride-based optoelectronic devices [46]. Due to higher tolerance to the lattice mismatch, dislocation-free NWs can be grown on different 
substrates, strongly reducing the production costs, and heterostructures can be incorporated maintaining the high quality crystal structure [7-9]. This is particularly advantageous in GaN NWs, where a defectfree structures can be achieved with a possibility to create heterostructures without affecting the material quality, which is particularly useful for devices such as LEDs, photodetectors and resonant tunnelling devices [8-10].

These advantages of NWs can be exploited for the design of nanostructured GaN UV-photodetectors. Generally, wide-band gap semiconductors present a range of advantages with respect to the standard materials used in commercial UV detectors (which are typically based on Si). First, they are transparent in the IR and visible range, eliminating the need of filters. Second, their direct bandgap ensures a high sensitivity. They can also be operated in challenging environmental conditions thanks to the material thermal stability and the ageing-resistant properties [11,12]. GaN NWs have been extensively studied for photo-detection application in the UV range $[13,14]$. In the last years, it has been demonstrated that insertion of high resistance heterostructures, as AlN barriers, in n-doped GaN NWs can boost the performances by reducing the dark current and enhancing the photosensitivity $[10,15]$. To reduce the dark current while keeping the efficient collection of the photo-generated carriers it is more advantageous to introduce multiple GaN/AlN insertions than a single thick AlN barrier. The improvement of the detection properties in GaN NWs containing AlN/GaN multiple quantum discs insertions compared to bare GaN NWs has been experimentally demonstrated in previous works [16].

However, due to the nanoscale dimensions of the NWs, the macroscopic characterization of the device properties offers a poor description of the physical phenomena occurring at a single NW level since standard characterization techniques probe millions of wires at the same time. Electron beam probe techniques can be used to investigate the properties of individual NWs [17-19] and to get an insight into the phenomena ruling the photodetection properties at the nanoscale.

In this work, we investigate individual GaN NWs containing 20 periods of AlN/GaN quantum discs (MQDs region) by electron beam induced current microscopy (EBIC) and cathodoluminescence (CL). The parameters extracted from structural analyses were used to perform a numerical simulation of the band profile along the wire axis with NextNano ${ }^{\circledR}$ software, which predicts the existence of two regions with opposite internal electric fields in these wires. A recent publication pointed out that the existence of these two fields reduces the sensitivity of the photodetector when operating without any external bias; nevertheless, due to the presence of two opposite fields, photo-detecting properties are exploited both under positive and negative bias, with comparable sensitivity [20]. In this work we clarify the charge collection mechanisms occurring at different bias investigating single NWs by in-situ I(V) measurements and EBIC mapping. A close correlation of the electrical properties to the structure of the NWs is obtained as well. Optical characterization is performed by low temperature $\mu$-PL and CL on dispersed NWs, revealing a blue-shifted GaN peak in the bottom segment of the wires due to strain. Moreover, a reduction of the luminescence is observed in the region where the electric fields are effectively splitting the generated charges. 


\section{Nanowire growth and structural analysis}

The n-i-n GaN/AlN axial heterostructure NWs were grown on $\mathrm{Si}(111)$ substrates in a plasma-assisted molecular beam epitaxy (PAMBE) chamber, active nitrogen being supplied by a radio-frequency plasma cell. The substrate temperature was fixed at $800^{\circ} \mathrm{C}$ and the growth was performed under $\mathrm{N}$-rich condition with a N/III ratio of about 1.36 for GaN and of about 9 for AlN. Prior to the growth of GaN NWs, a 2.5$\mathrm{nm}$-thick AlN buffer layer was deposited on the substrate [21]. 20 periods of multiple GaN/AlN discs (MQDs) were integrated in the non-intentionally-doped active region of the NW volume, while the GaN base and GaN-cap NW parts were n-doped with $\mathrm{Si}\left(\mathrm{T}_{\mathrm{Si}}=1200^{\circ} \mathrm{C}\right)$. The NWs formed under these conditions are vertically aligned with hexagonal shape delimited by $\{10-10\}$ planes [22].

The n-i-n GaN/AlN heterostructured NWs have been analysed by scanning transmission electronic microscopy (STEM), using a $200 \mathrm{keV}$ FEI Titan Themis system equipped with a probe aberration corrector and a super-X Bruker silicon drift detector (effective angle of detection of 0.98 steradian). The measurements have been performed on NWs detached from their substrate and dispersed on a $\mathrm{Cu}$ grid covered by a carbon membrane. The STEM images clearly show the three characteristic segments present in these wires: the $\mathrm{n}-\mathrm{GaN}$ stem, the AlN barriers/GaN discs region and the $\mathrm{n}-\mathrm{GaN}$ cap (Figure 1a). The $\mathrm{GaN}$ discs and the AlN spacers are characterized by abrupt interfaces and have been measured to be respectively $\sim 0.6$ and $4.5-7 \mathrm{~nm}$ thick (Figure $1 \mathrm{~b}$ ). In fact, some NWs present AIN spacer widening with stacking, then inducing a continuous NW enlargement with a consequent thinning of the barriers along the growth direction. The NWs enlargement is associated with the formation of unintentional AlN/GaN radial multilayers surrounding the MQDs region and the GaN NW base. The structural analysis showed that together with the axial growth of the AlN barriers, a non-intended radial growth occurs, which results in the formation of a continuous AIN shell around the $n-G a N$ base [23]. Moreover, an additional outer radial layer of $\mathrm{GaN}$ can be formed during the GaN cap growth. The formation of unintentional shell around the bottom part of the NW during the axial heterostructure growth has been already reported in the literature, its appearance being dependent on the local NW environment and shadow effects [16,24]. As an example, EDX maps in Figure 1c show the case of a NW with an inner/outer shell (of AlN and GaN, respectively) around the NW stem. From STEM images, the thickness of the GaN layer in the shell can vary from $1 \mathrm{~nm}$ to several tens of nanometers. Depending on the thickness of the parasitic shell, the electrical properties at a single NW level can be very different, thus affecting the performance of optoelectronic devices at the macroscopic scale. Indeed, the thickness of the shell has an influence on the conductivity of the NWs. Due to Fermi level pinning at the surface, thin shells could be completely depleted, hence giving no contribution to the overall conductivity of the NW [25]. On the other hand, thick GaN shells would offer a low resistance path to the carriers, thus increasing the dark current level and decreasing the photosensitivity factor. To distinguish these extreme cases, two nominal structures are considered in the following: GaN NWs with a parasitic AlN shell without any GaN, labelled as " $N W$ $\boldsymbol{A}$ ” (Figure 1d) and GaN NWs with a parasitic AlN/GaN shell, labelled as " $\boldsymbol{N} \boldsymbol{W} \boldsymbol{B}$ ” (Figure 1e). 

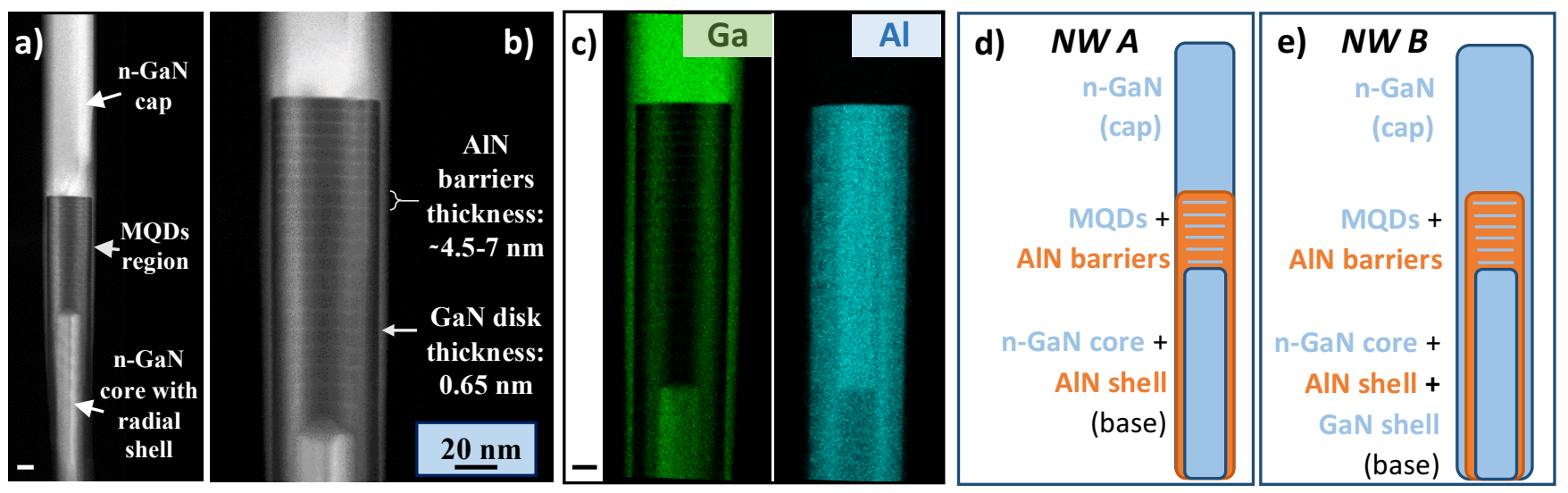

Figure 1. a) STEM image of a single GaN/AIN NW (the scale bar corresponds to $20 \mathrm{~nm}$ ); b) STEM image of the multiple quantum discs region embedded in a GaN NW; c) EDX maps of a single NW for Ga and Al atoms (the scale bar corresponds to $10 \mathrm{~nm}$ ); Schematic internal structure of a nanowire (d) with bottom AlN shell, labelled as $\mathbf{N W} \boldsymbol{A}$ and (e) with bottom GaN/AlN shell, labelled as $\mathbf{N W} \boldsymbol{B}$.

\section{Investigation of the charge collection properties}

\subsection{EBIC mapping and single I(V) characteristics}

The electrical properties of individual NWs were investigated by single NWs I(V) measurements and EBIC microscopy. For this purpose, the NWs were dispersed on a $\mathrm{Si}$ wafer with a $300 \mathrm{~nm}$ thermal $\mathrm{SiO}_{2}$ layer on top and individually connected to metal pads (Figure 2a). After the dispersion, the NWs with a length of $\sim 1.5 \mu \mathrm{m}$ were selected, and the metal contacts to the top and the bottom part of the NWs were designed and fabricated by electron beam lithography technique. In order to obtain an ohmic contact on the $\mathrm{n}-\mathrm{GaN}$ NWs, a Ti/Al/Ti/Au (5/25/15/100 nm) metal deposition is used [26]. SEM contrast allows to localize the MQDs region (being rich in Al, it appears darker as highlighted by the red circle in Figure 2a), which in most of the cases is found to be close to the contact (or in some cases even partially buried under the contact). In EBIC microscopy electron-hole pairs are locally generated by the electron beam. In structures with an internal electric field, the electron-hole pairs created within or close to the field region can be separated and collected producing a measurable current [18,27,28]. 

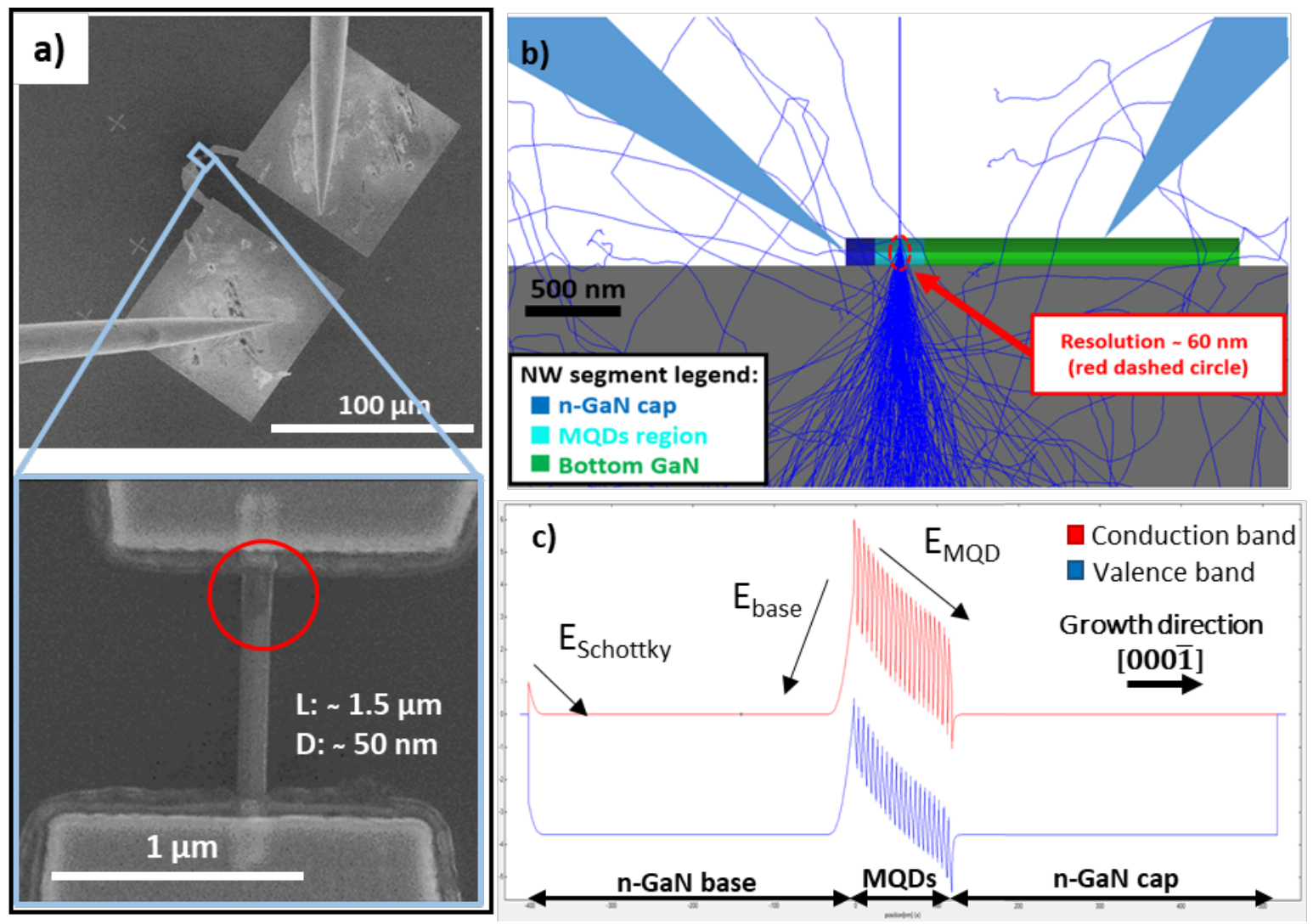

Figure 2: a) Low magnification SE image of the metal pads connected to single NWs, with nanomanipulators on the contact pads and high magnification SE image of a single NW with contacts. The red circle highlights the $M Q D$ s region. b) Monte Carlo simulation with Casino software of the electron beam/NW interaction volume at the EBIC operational conditions. The blue lines represent the trajectories of the electrons from the beam. In the inset, $N W$ segment colour legend. c) NextNano $($ simulation of the axial band profile.

EBIC was performed in the Hitachi SU-8000 SEM using Kleindiek PS4 nanomanipulators equipped with tungsten tips to connect metal pads to the external circuit. An external bias ranging from $-4 \mathrm{~V}$ to +4 $\mathrm{V}$ was applied through a Stanford SR560 preamplifier controlled by a Gatan DigiScan system. The external bias is used to increase or flatten selectively different electric fields within the NWs. The same setup was used to measure single NW I(V) curves, acquired in-situ by means of a Keithley 2636 sourcemeter, coupled with a LabView acquisition system. More details about the set-up can be found in previous works [17,18,29]. Electron beam was set at $25 \mathrm{kV}$ beam acceleration voltage and $145 \mathrm{pA}$ injected current. These beam conditions were chosen to optimize both EBIC spatial resolution (which is estimated $\sim 60 \mathrm{~nm}$ by the Monte Carlo simulation, as displayed in Fig. 2b), and the corresponding secondary electron (SE) image. Indeed, due to the small diameter of the NWs $(\sim 50 \mathrm{~nm})$ the use of a high acceleration voltage allows improving the resolution since the interaction volume exits the $\mathrm{NW}$ and broadens further down in the substrate (Fig. 2b)).

Before the measurements, the axial band profile of the NWs is simulated by means of the NextNano ${ }^{\circ}$ software using the geometrical parameters gathered from the previously described structural analysis (Figure 2c). The calculation was performed in 1D approximation, i.e. neglecting the radial band bending effects. This rough approximation cannot provide the quantitative information on the confinement in the quantum discs, however it evidences the existence of regions containing a built-in field. Indeed, the 
profile shows the presence of two internal fields oriented in opposite direction, respectively across the MQDs region $\left(\mathrm{E}_{\mathrm{MQD}}\right)$ and within the $\mathrm{n}-\mathrm{GaN}$ base $\left(\mathrm{E}_{\text {base }}\right)$. In addition, a Schottky barrier is expected at the interface between the metal contact and the bottom end of $\boldsymbol{N} \boldsymbol{W} \boldsymbol{s}$, due to the presence of the outer AlN shell. The built-in field of this Schottky barrier ( $\left.E_{\text {Schottky }}\right)$ is oriented in the same direction as the electric field induced in the MQDs region and opposite to the field in the GaN base (as shown in Figure 2b).

In the following, we investigate the influence of this complex internal electric field distribution on the carrier collection at a single NW level in order to help clarifying the electrical properties of these structures. For all the measurements described in the following, the top GaN cap is connected to the positive pole of the source-meter while the bottom segment is connected to the negative pole.

Among the tested NWs, two main groups have been identified by their I(V) characteristics and their EBIC maps. The observed properties can be ascribed to the two categories of structures previously described as $\boldsymbol{N} \boldsymbol{W} \boldsymbol{A}$ and $\boldsymbol{N} \boldsymbol{W} \boldsymbol{B}$. In the following, electrical characterizations of one representative NW from each category are described.
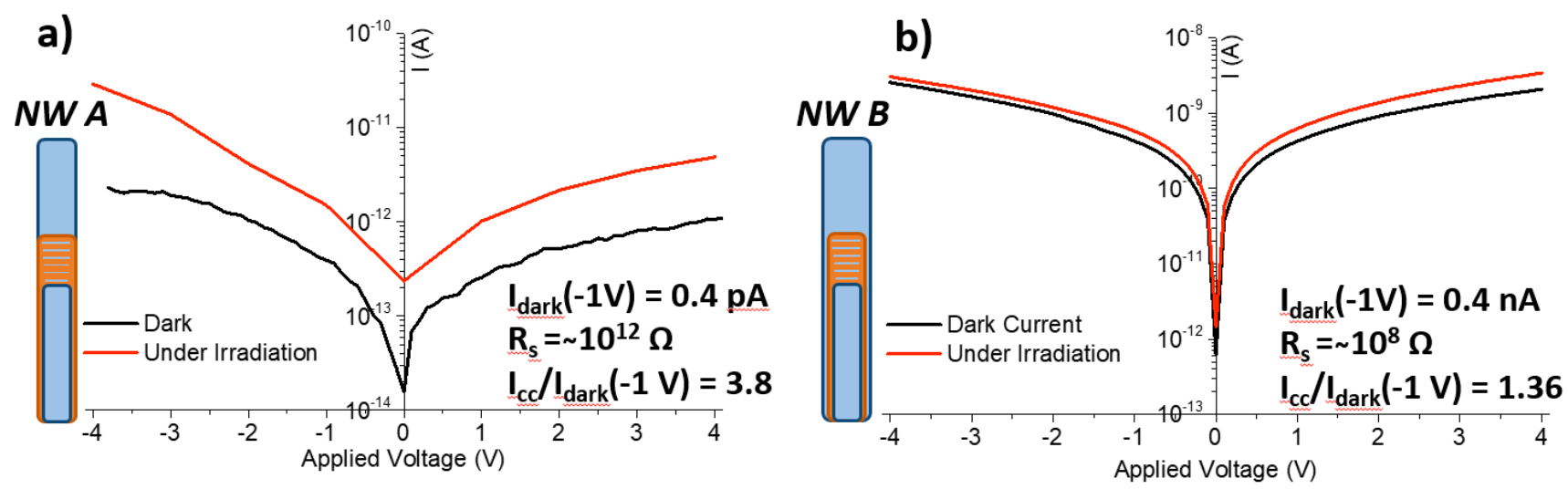

Figure 3: Single NW I(V) characteristics in dark (black curve) and under electron beam irradiation conditions (red curve) of NWA (a) and of NW B (b). The insets give the parameters representative of the electrical properties of each structure.

An example of I(V) characteristics (in dark and under electron beam irradiation) for a single $\boldsymbol{N} \boldsymbol{W} \boldsymbol{A}$ is shown in Figure 3a. In dark conditions the NW shows an I(V) curve (black curve) characterized by a very high series resistance $\left(R_{s}\right.$, estimated in the order of $10^{12} \Omega$ ) and a dark current value ( $\left.I_{\text {dark }}\right)$ below 1 $\mathrm{pA}$ at $-1 \mathrm{~V}$. By using the electron beam as an irradiation source, the induced current $\left(\mathrm{I}_{\mathrm{cc}}\right)$ has been collected as a function of the external voltage.

Although in these conditions no information can be gathered on the photon detection properties of the NWs, the electron beam irradiation can give an idea of the variation of the current collected under excitation conditions. To quantify this property, we define the electron beam sensitivity factor as $\mathrm{I}_{\mathrm{cc}} / \mathrm{I}_{\mathrm{dark}}(\mathrm{V})$, analogously to the photosensitivity factor defined as $\mathrm{I}_{\mathrm{ph}} / \mathrm{I}_{\mathrm{dark}}(\mathrm{V})$, where $\mathrm{I}_{\mathrm{ph}}$ indicates the current collected under light illumination. In these conditions, $\boldsymbol{N} \boldsymbol{W} \boldsymbol{A}$ demonstrates an induced current sensitivity of $\sim 4$ under a bias of $-1 \mathrm{~V}$, as reported in Figure 3a. This measurement shows a low value with respect to the photosensitivity factors reported in the literature under UV light illumination [16]. However, since an electron beam irradiation is used in the present work a direct comparison on the charge collection properties cannot be done.

I(V) characteristics for the $\boldsymbol{N} \boldsymbol{W} \boldsymbol{B}$ (shown in Figure 3b) indicate a significantly different electrical response with respect to the $\boldsymbol{N} \boldsymbol{W} \boldsymbol{A}$. The I(V) curve in dark shows a diodic behavior characterized by a 
higher conductivity as highlighted by the $I_{\text {dark }}(-1 \mathrm{~V})=0.4 \mathrm{nA}$ and the $\mathrm{R}_{\mathrm{s}}$ value around $10^{8} \Omega$. The latter parameter is estimated by fitting the characteristic curve with a one-diode model. In this case, the electron beam sensitivity factor $I_{c c} / I_{\text {dark }}(-1 \mathrm{~V})$ is very close to 1 , indicating a smaller percentage variation of the collected current under electron beam irradiation than what was observed for the $\boldsymbol{N} \boldsymbol{W} \boldsymbol{A}$. However, it is worthy to notice that under the same excitation conditions, the photo-current measured in $N \boldsymbol{W} B$ is higher than in $\boldsymbol{N} \boldsymbol{W} \boldsymbol{A}$. This indicates that, also due to the lower resistance, the NW B is more sensitive to the external excitation. These results are coherent with reports in the literature which indicate a resistance value higher than $10^{7} \Omega$ and a photosensitivity factor lower than 2 (under UV illumination at $\lambda=300 \mathrm{~nm}$ with a power density of $5 \mathrm{~mW} / \mathrm{cm}^{2}$ ) in wires with a parasitic AlN/GaN bottom shell [16].

An insight on the influence of the internal electric field on the described electrical properties is obtained by performing EBIC mapping on the same nano-objects at different external bias (Figure 4). The EBIC analyses discussed in the following allow to precisely locate the current generation within the wire under different bias conditions and give the first direct evidence of carrier collection from AlN/GaN MQDs. Concerning $\boldsymbol{N} \boldsymbol{W} \boldsymbol{A}$ (Figure 4a), under forward bias $(\mathrm{V}>0)$, an electrical signal is collected, highly localized at the bottom NW/contact interface, indicating the presence of a Schottky barrier between the deposited metal and the base segment of the $N \boldsymbol{W} \boldsymbol{A}$. The formation of a Schottky contact was expected due to the presence of a parasitic AlN outer shell on the lower part of the wire, as depicted in Figure $1 \mathrm{~b}$. The absence of an induced current corresponding to the MQDs region is surprising. However, it is worthy noticing that the high resistance of the wire decreases the current sensitivity of the measurements, therefore weak electric fields could be hard to visualize in these conditions. In addition, the presence of $E_{\text {Schottky }}$ oriented in the same direction as the electric field induced by the MQDs region may lead to an inhomogeneous voltage drop along the wire, which hinders the current collection from one of the regions.

The same reasons could be responsible for the lack of electrical signal when EBIC mapping is performed without external bias $(0 \mathrm{~V})$. Under reverse bias, instead, an electrical signal from the GaN base appears due to the flattening of the band profile in the Schottky region. In this case, the induced collected current is well below $1 \mathrm{nA}$ as a result of a highly resistive nature of the wire. However, the direction of the induced current is coherent with the electric field expected in the GaN base, revealing that the excess carriers are drifted apart by $\mathrm{E}_{\text {base }}$ when a negative external bias is applied.

These results enable to determine the charge collection mechanisms in single NWs. In fact, referring to the corresponding I(V) curve (reported in Figure $4 \mathrm{~b}$ for clarity), we can attribute the photo-detection properties to the charge splitting occurring in the GaN base of the structure under reverse bias and the induced current collected under a forward bias to the electric fields arising at the Schottky contact and/or in the MQDs region. Although it is possible to have a qualitative information by comparing EBIC maps and I(V) curves, a quantitative comparison is not possible due to the different data acquisition in the two cases. Moreover, in the case of $\boldsymbol{N W} \boldsymbol{A}$ the high resistance is a limiting factor since a sub-nA current is collected, as previously pointed out.

With this regard, the higher conductivity of NW B can be beneficial for a deeper analysis of the EBIC maps. In fact, the presence of the parasitic outer AlN/GaN shell does not modify the electronic structure of the inner core. By reducing the resistance, it offers an electrical access allowing a more detailed investigation of the internal electric fields. 

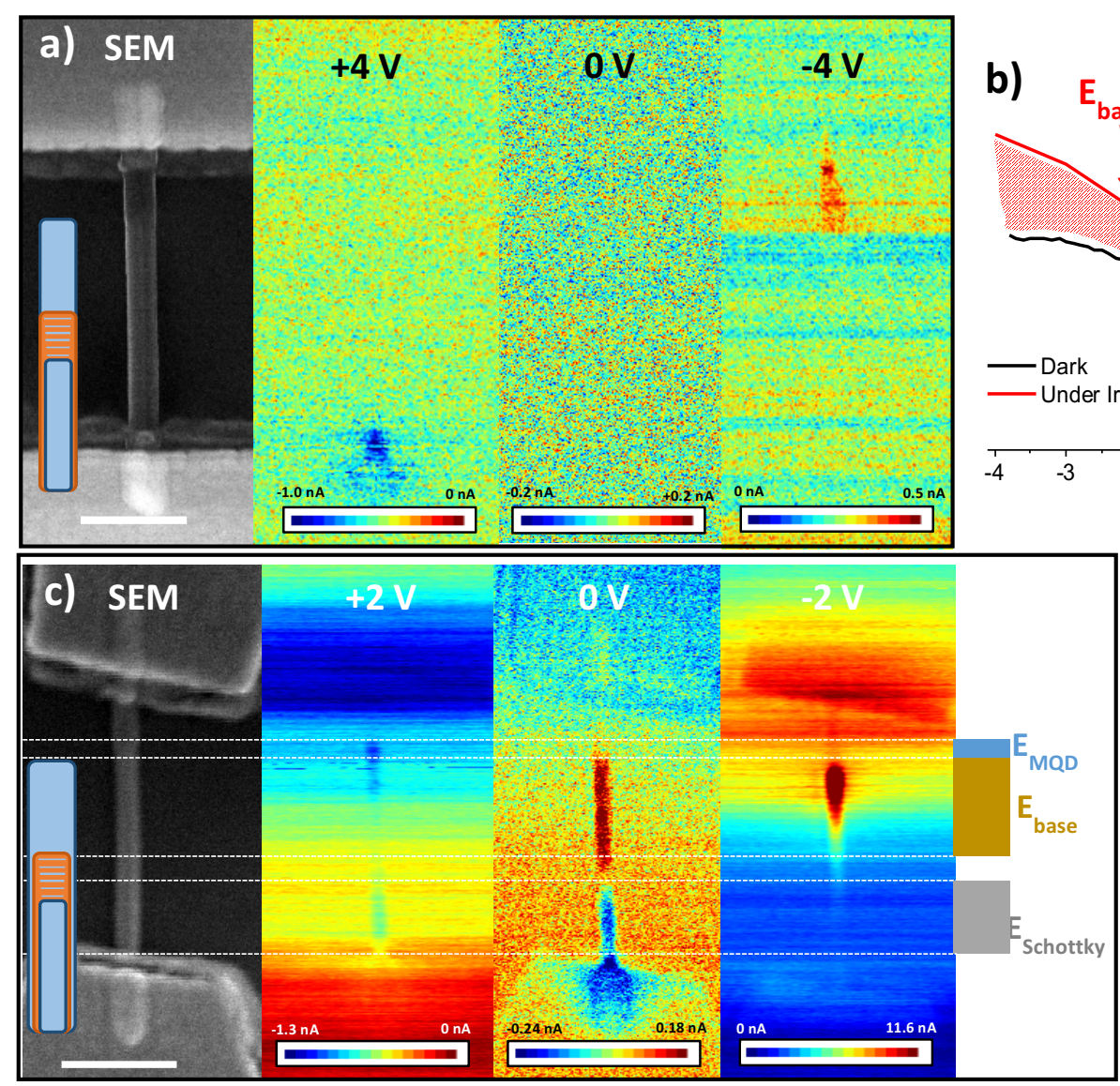
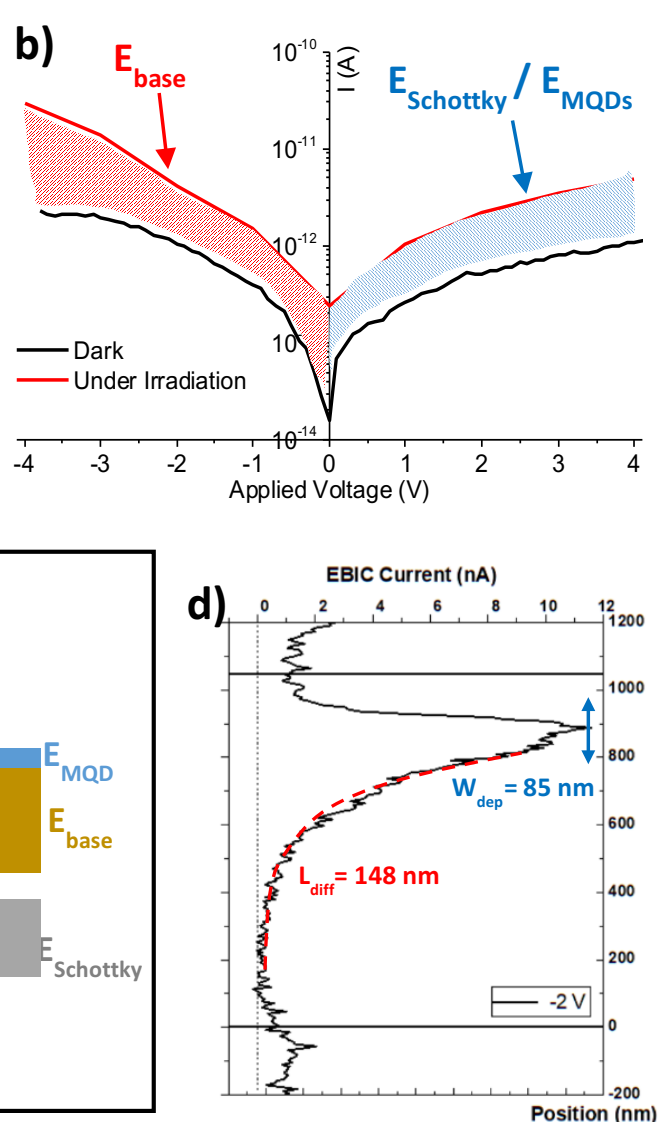

Figure 4: a) SE image (the scale bar corresponds to $0.5 \mu \mathrm{m}$ ) and corresponding EBIC maps at different bias for $N W A$; b) I $(V)$ curves in dark and under electron beam irradiation for $N W A$. The red and blue lines between curves highlight the effect of the internal electric fields on the collected current in the different voltage ranges. $c$ ) $S E$ image (the scale bar corresponds to $0.5 \mu \mathrm{m}$ ) and corresponding EBIC maps at different bias for $N W B$. The white dashed lines indicate the regions of activity of the different internal electric fields, indicated in the legend aside. d) EBIC profile along the central axis of the $\mathrm{NW}$ extracted from the map acquired at $-2 \mathrm{~V}$.

The EBIC maps of $\boldsymbol{N W} \boldsymbol{B}$ at different bias, reported in Figure 4c, allowed to observe the activity of all the regions containing the internal electric fields - namely, $\mathrm{E}_{\mathrm{MQD}}, \mathrm{E}_{\mathrm{base}}$ and $\mathrm{E}_{\text {Schottky. Under forward }}$ bias $(+2 \mathrm{~V})$ the induced current is collected from both the MQDs region and the Schottky contact, as predicted from the previously discussed band profile. As expected, the collection of charges within the MQDs region occurs in a narrow section estimated to be around $190 \mathrm{~nm}$. This estimation matches the expected extension for the charge collection volume across the MQDs region which extends for $120 \mathrm{~nm}$. Considering the EBIC map resolution of $60 \mathrm{~nm}$, the EBIC signal for the MQDs was expected to be around $180 \mathrm{~nm}$ wide. No further carrier diffusion is expected since the field $\mathrm{E}_{\mathrm{MQD}}$ is confined between the base (where the field $E_{\text {base }}$ has an opposite direction) and the triangular potential well at the GaN cap/AlN barrier interface appearing due to the difference between the spontaneous polarizations of GaN and AlN. The signal collected from the Schottky contact on the bottom part of the wire can be better visualized in EBIC maps without applied bias. In the $0 \mathrm{~V}$ map, it can be observed that the Schottky signal is maximum at the NW/contact interface, but it extends above and below the contact area. In addition, in these conditions the current flowing in the opposite direction, arising from the $\mathrm{E}_{\text {base }}$ field in the GaN base, is observed along a large segment $(\sim 600 \mathrm{~nm})$, which is positioned between the MQDs region and the 
Schottky contact. As expected under reverse bias $(-2 \mathrm{~V})$ the signal collected from the GaN base becomes predominant and an induced current up to $\sim 12 \mathrm{nA}$ can be collected. The weaker signal in the MQDs compared to the NW base may be due to a strong carrier localization in the quantum discs due to high AlN confining barriers, which prevents efficient carrier extraction. Previously, photocurrent arising from charge splitting in MQDs was reported under optical pumping [19]. It was indirectly identified as arising from the MQDs thanks to a spectral shift with respect to the GaN bandgap. Thanks to EBIC technique used in the present study, the location of this signal can be directly visualized allowing to attribute the induced current to the MQD region.

To quantitatively analyse the variation of the induced current in the GaN core, the EBIC current profile was extracted along the central axis of the NW (Figure 4d). The EBIC profile shows that under reverse bias, the induced current abruptly increases at the MQDs/GaN base interface (the estimated minority carrier diffusion length across this interface is $25 \mathrm{~nm}$ ) and then is constant in a region $\sim 85 \mathrm{~nm}$ wide. This is the extension of space charge region $\left(\mathrm{W}_{\mathrm{dep}}\right)$ induced by $\mathrm{E}_{\mathrm{base}}$, which drives the charge collection when $\mathrm{V}<0$. In addition, charges are collected also due to diffusive transport phenomena occurring in the lower part of the segment. By fitting the EBIC profile with an exponentially decaying function, it has been possible to estimate the hole diffusion length in the $\mathrm{n}-\mathrm{GaN}$ core around $148 \mathrm{~nm}$, similarly to other reports in the literature for single crystalline $\mathrm{n}-\mathrm{GaN}$ wires with an estimated residual doping $10^{17} \mathrm{~cm}^{-3}[30]$.

\subsection{Photoluminescence spectra and cathodoluminescence mapping}

Since EBIC analysis indicates that these wires have good transport properties due to their crystalline perfection, the optical properties of the NWs were addressed by PL spectroscopy. PL spectra were acquired at $4 \mathrm{~K}$, the excitation being provided by $244 \mathrm{~nm}$ light emitted by a continuous wave frequency doubled Argon ion laser and focused on a spot of the size of around $3.5 \mu \mathrm{m}$ at an incident power density of $100 \mathrm{~W} / \mathrm{cm}^{2}$ ). The generated PL signal was dispersed by a 1800 grooves per mm grating in a $460 \mathrm{~nm}$ spectrometer allowing for a spectral resolution of around $0.25 \mathrm{meV}$ and collected by a LN2-cooled silicon charged-coupled device (CCD). PL measurements were performed on individual NWs dispersed on a $\mathrm{Si} / \mathrm{SiO}_{2}$ substrate. An example of a representative spectrum is given in Figure 5.

Spectra show GaN donor bound exciton $\left(\mathrm{D}^{0} \mathrm{X}_{\mathrm{A}}\right)$ emission peak at $3.476 \mathrm{eV}$ for all the investigated NWs, together with a shoulder at $3.454 \mathrm{eV}$ and a lower intensity peak at $3.369 \mathrm{eV}$, respectively associated to inversed domain boundary emission and stacking faults (SFs) transitions [31,32]. A large number of peaks with higher energy is found with different recurrence among the investigated NWs. A large number of NWs ( $60 \%)$ show a pronounced peak in the $3.564-3.626 \mathrm{eV}$ range. In all the observed cases, the full width at half maximum (FWHM) of these peaks is from 4 to $30 \mathrm{meV}$ larger than for the emission associated to the donor bound exciton. Such blue-shifted peaks were previously reported in GaN NWs compressively strained with an AlN shell [33-35]. We attribute this spectral contribution to the NW base part surrounded with the AIN shell. The variation of the shell thickness from wire to wire explains the energy difference between the observed peaks. The increased broadening is ascribed to the shell inhomogeneity leading to a variation of strain. 


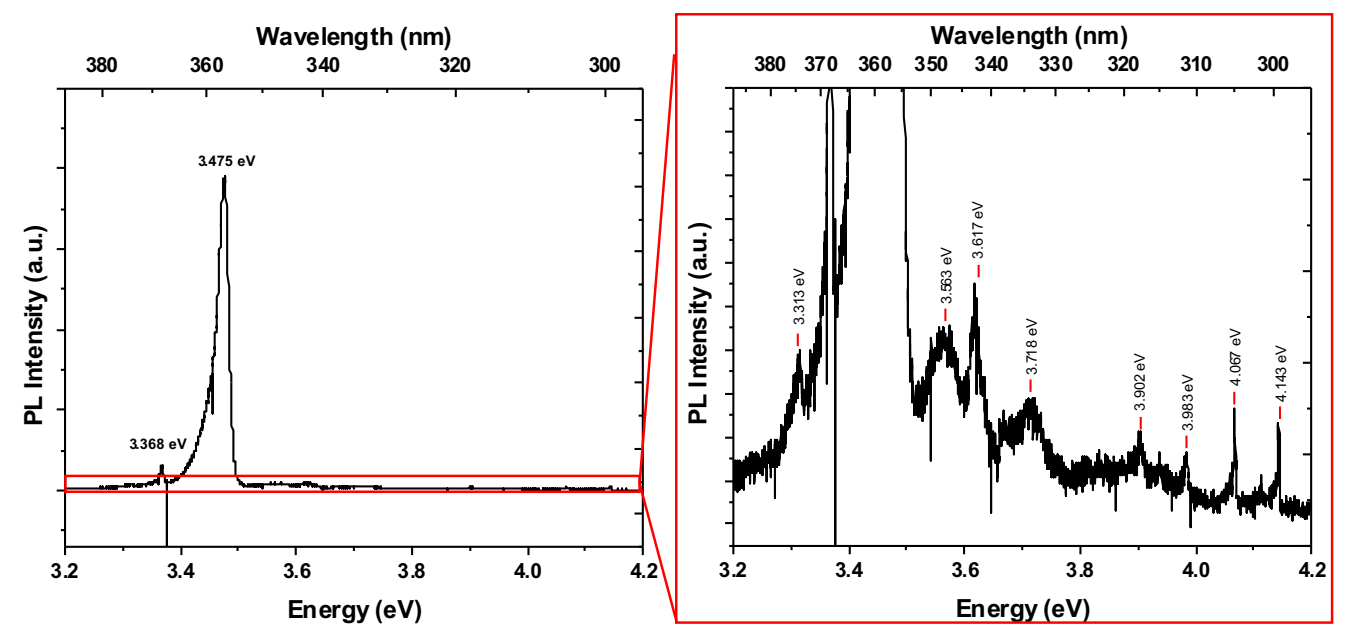

Figure 5: Example of PL spectra of AlN/GaN axial heterostructure NWs. Magnification is shown within the red square.

In addition, several signals are found with a peak wavelength lower than $325 \mathrm{~nm}$, i.e. characterized by a peak energy higher than $3.8 \mathrm{eV}$. Their presence and spectral position is not systematic in all the NWs (their recurrence is lower than 25\%). Both observations suggest that they could be related to the $\mathrm{GaN}$ discs in the QDs region or to the GaN/AlN structures in the parasitic radial multi-layer [34]. For both of them a high energy emission is expected due to the quantum confinement with a peak wavelength strongly dependent on the local insertion morphology.

To get an insight on the spatial distribution of these high energy emission peaks in single NWs, CL analyses were performed on individual NWs at $15 \mathrm{~K}$. In CL measurements, the photons emitted due to radiative recombination events can be associated to the excitation electron beam position hence building a hyper-spectral map where a spectrum is associated to each pixel. By integrating the luminescence intensity associated to each pixel in different wavelength ranges, it is possible to obtain spectrally filtered maps of individual NWs [36-38]. The measurements were performed with an Attolight "Chronos" cathodoluminescence microscope with an acceleration voltage of $2 \mathrm{kV}$ and the spectra were recorded on an Andor Newton CCD camera with a Horiba dispersive spectrometer using a 150 grooves $/ \mathrm{mm}$ grating. Light is collected through an achromatic reflective objective with a numerical aperture of 0.7. The measurements were firstly performed on the same nano-objects analysed by EBIC mapping. Unfortunately, no clear results were obtained since the MQDs is partially covered with the metal contact. Moreover, the optical emission of these wires was degraded after several hours of EBIC observations yielding a low CL signal and a poor signal to noise ratio. For this reason, CL maps of fresh NWs not exposed to the electron beam were analyzed and are presented in the following. 

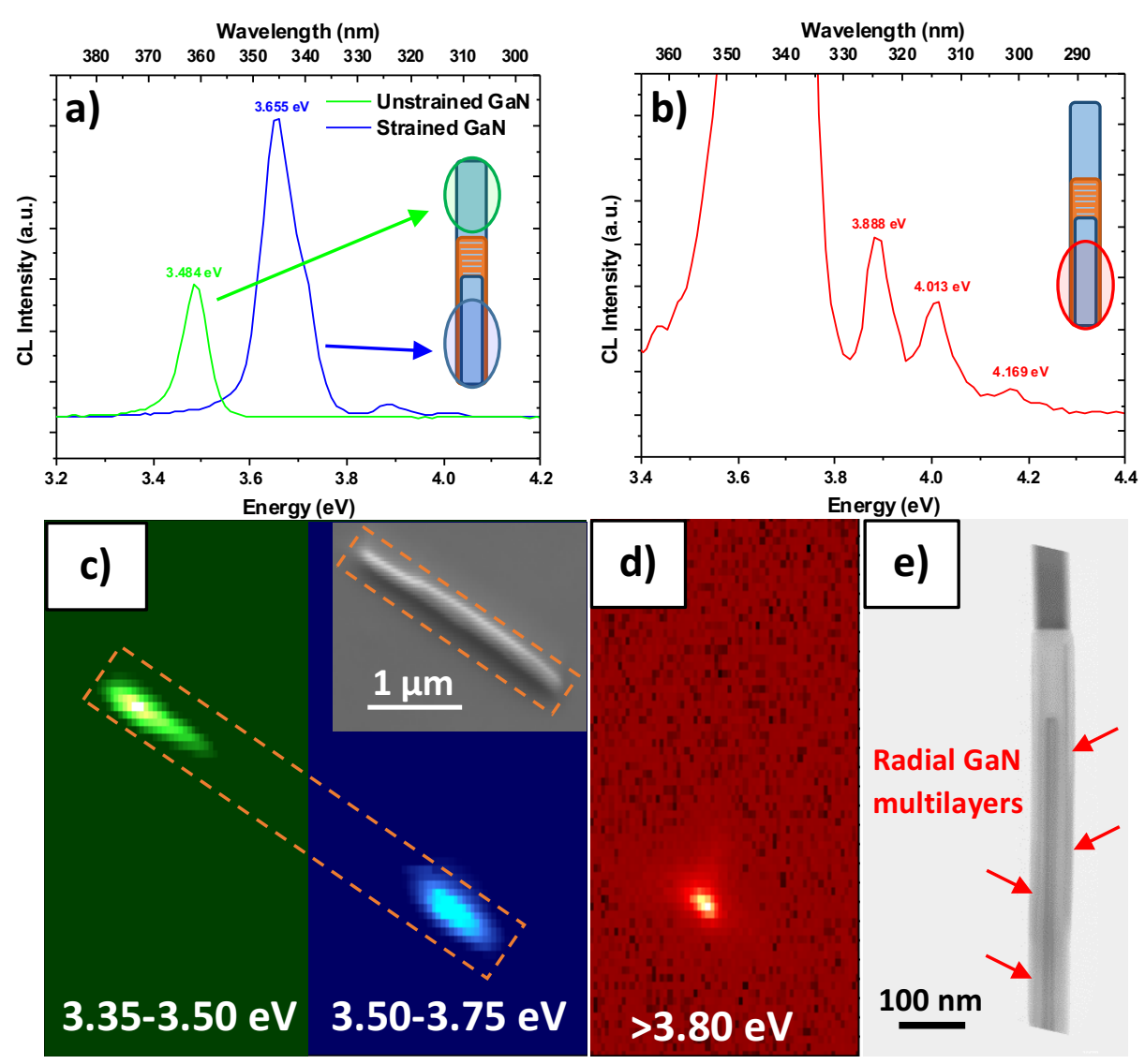

Figure 6: Low temperature CL analysis. Luminescence spectra emitted at different positions showing the peaks attributed to unstrained and strained $G a N D^{\circ} X_{A}$ transition (a) and to GaN discs or radial stacking (b). The insets represents schematically the localization of the signal showed in panels $c$ and $d$. c) CL maps of a single NW showing the emission of unstrained GaN (3.35-3.50 eV) and strained GaN (3.50-3.75 eV). In the inset, a SE image of the investigated wire. d) CL map filtered for wavelength higher than $3.80 \mathrm{eV}$; e) STEM image showing radial GaN insertions embedded in the AlN parasitic shell.

Coherently with the PL analysis, the CL measurements show luminescence spectra characterized by the presence of the unstrained and strained GaN peaks, respectively at $3.484 \mathrm{eV}$ and $3.655 \mathrm{eV}$, emitted from the extremities of all the investigated NWs (Figure 6a). Also high energy peaks with different spectral position ranging from $290 \mathrm{~nm}$ to $319 \mathrm{~nm}$ (i.e. with an energy peak between $3.89 \mathrm{eV}$ and 4.28 $\mathrm{eV}$ ) are observed as displayed in Figure $6 \mathrm{~b}$.

In order to identify the origin of these different emissions, three CL maps are built by integrating the luminescence in the range of 3.35-3.5 eV, of 3.5-3.75 eV and above $3.8 \mathrm{eV}$. An example is given in Figures $6 \mathrm{c}$ and $\mathrm{d}$, together with the SE image of the investigated NW (in the inset).

The CL maps reveal that the GaN emissions are highly localized at the extremities of the wire. In particular, the unstrained $\mathrm{GaN}$ luminescence is emitted from the top shell-free segment, while this emission is blue-shifted in the bottom segment due to the presence of the parasitic radial shell.

CL maps filtered for photon energies higher than $3.8 \mathrm{eV}$ show that in few wires $(\sim 25 \%)$ a localized high energy luminescence emitted is present in the bottom segment of the wire (Figure 6d). Although the resolution of the CL maps is not sufficient to determine the exact origin of these peaks, their localization in the bottom segment allows to refute their attribution to the MQDs. We attribute these emissions to 
lateral GaN/AlN structures formed in the parasitic shell. Indeed, the presence of these parasitic GaN insertions was evidenced in the bottom shell by STEM analysis (Figure 6e). These insertions, embedded in the AlN shell, are probably created due to radial GaN growth during the discs formation, as reported in the literature $[24,34,35,39]$. The attribution of the high energy emission to the non-intentional GaN radial insertions is also consistent with the PL results; it is likely that the uncontrolled growth of these structures is the origin of the large dispersion from NW to NW of the peak wavelengths associated to the high energy emissions, as discussed in Figure 5. The higher intensity of the luminescence arising from the non-intentional $\mathrm{GaN}$ radial insertions compared to the axial MQDs may be due to their non-polar confining direction.

One open question concerns the radiative recombination in the MQDs region, where high energy peaks with an energy around 4.2-4.4 eV are expected from $0.65 \mathrm{~nm}$ thick QDiscs [40,41]. These emissions were not observed in this study since, interestingly, in all analyzed NWs a large region with very low intensity emission is observed in part of the wire, where the MQDs are located. To understand the origin of the reduction of the luminescence intensity in the central NW part, the CL panchromatic map (i.e. spectrally integrated map) is analyzed (Figure 7a). This map shows the intensity integrated over the entire detected spectral range $(125-670 \mathrm{~nm})$ evidencing that the CL signal in the middle part of the wire drops for any wavelength. By extracting the intensity profiles along the wire axis from the CL maps, this luminescence drop can be clearly observed in the segment corresponding to the MQDs region and the adjacent part of the GaN stem (Figure 7b). By comparing this profile to the EBIC maps described in the previous Section, a strong anti-correlation is found, indicating that the charge drift observed in EBIC may be the cause of the decrease of luminescence in the central segment. Referring to the schematic of the NW in Figure 7b, it can be observed that CL intensity decreases in the segments where the internal electric fields drift apart the generated carriers. As a confirmation of this hypothesis, the segment with low CL intensity is estimated to be around $720 \mathrm{~nm}$ long, coherently with EBIC maps which show a 200 $\mathrm{nm}$ wide electrical signal induced in the MQDs region and a $580 \mathrm{~nm}$ wide induced current in the GaN bottom segment.
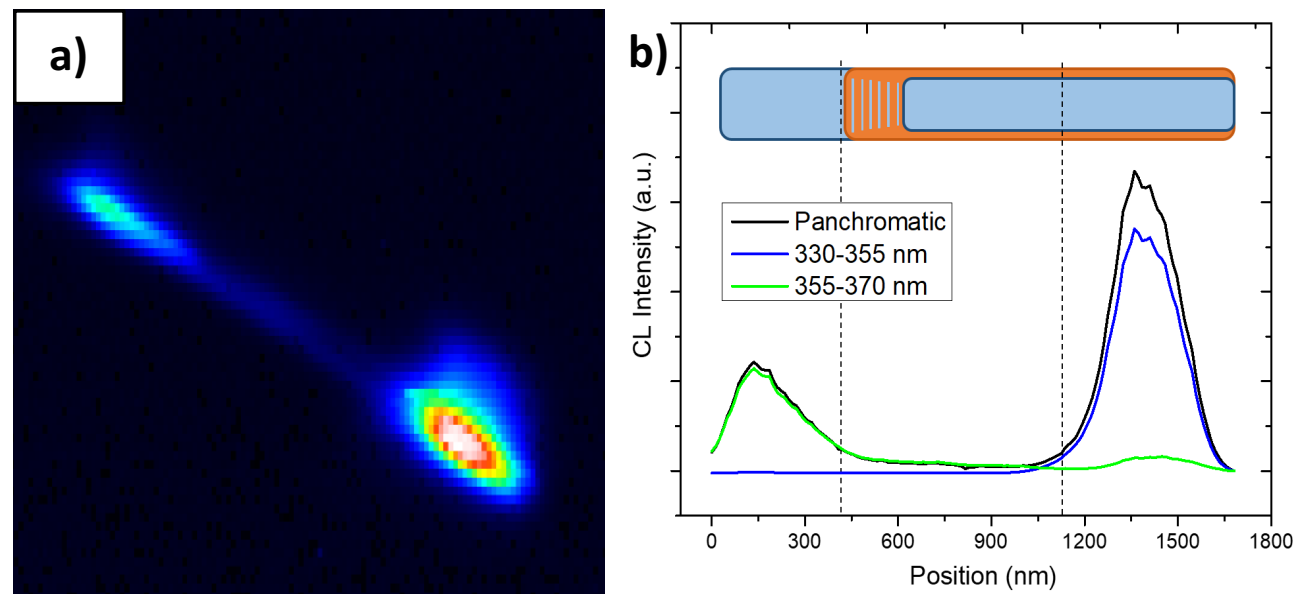

Figure 7: a) Panchromatic CL maps showing the luminescence variation along the wire; $b$ ) CL intensity profiles along the main axis of the $N W$ extracted from the panchromatic CL map (black curve) and from the CL maps filtered in the ranges of 330-355 $\mathrm{nm}$ and 355-370 $\mathrm{nm}$ (blue and green curves, respectively). A schematic of the NW is added as a reference for the interpretation of the profiles. 


\section{Conclusions}

In this work, the charge collection properties of $n-G a N$ NWs containing GaN/AIN multiple quantum discs are investigated at the nanometric scale. The structural analysis performed by STEM revealed the presence of parasitic shells around the bottom segment made of AlN and GaN with thickness ranging from 1 to tens of $\mathrm{nm}$. I(V) characteristics on single dispersed wires in dark and under electron beam irradiation indicated that the thickness of the GaN outer shell strongly influences the electrical properties of the wires. Thin GaN shells do not contribute to the overall electrical properties and therefore these NWs are characterized by a high series resistance, attributed to the AIN insertions, and by the ability to generate a current under excitation which is several times higher than in dark. The wires with thicker GaN shell are characterized by a resistance $10^{3}-10^{4}$ times lower and an induced current comparable to the dark one. The charge collection properties are investigated by electron-beam induced current microscopy. EBIC maps on resistive wires offer a poor insight on the charge collection properties while, a better electrical access to the internal electric fields was achieved in NWs having a parasitic GaN/AIN radial shell. EBIC microscopy revealed the co-existence of two electric fields oriented in opposite directions which act on the generated carriers (as predicted by the band diagram simulation by NextNano ${ }^{\odot}$ software): an electric field acting in the MQDs region and an electric field acting in the bottom GaN part adjacent to the MQDs. Moreover, it was possible to identify the voltage ranges where the different electric fields act on the excess carriers, i.e. the charge collection mechanisms ruling the photodetection properties of these wires under UV illumination.

Finally, the NWs have been investigated also by PL and CL analyses at low temperature. Several peaks have been identified, the most prominent related to the band edge transition of both strained and unstrained GaN. CL mapping proved that the strained GaN emission originates from the bottom part of the NW due to the presence of the radial shell. Also the emission of high energy photons, attributed to GaN radial confining insertions, are observed in the bottom part of the wire. CL mapping revealed that a strong luminescence drop occurs in the central part of the NWs. Comparing the results obtained with $\mathrm{CL}$ and EBIC, the decrease of the luminescence intensity overlaps with the strong EBIC signal and is attributed to an efficient charge splitting due to the presence of the electric fields in the MQDs region and in the $\mathrm{GaN}$ base.

\section{Acknowledgements}

The authors acknowledge the financial support from the European Union's Horizon 2020 research and innovation program "NanoTandem" under grant agreement No 641023. The Attolight cathodoluminescence tool was funded by public grants supported by the Region Ile-de-France in the framework of C'Nano IdF (the nanoscience competence center of the Paris region), by the European Union (FEDER 2007-2013), and by the Labex GANEX (grant no. ANR-11-LABX-0014) and NanoSaclay (grant no. ANR-10-LABX-0035) as part of the

"Investissements d'Avenir" program managed by the French National Research Agency (ANR). 


\section{References}

[1] Krogstrup P, Jorgenses H I, Heiss M, Demichel O, Holm J V, Aagesen M, Nygard J and Fontcuberta i Morral A 2013 Single nanowire solar cells beyond Shockley-Queisser limit Nat. Photonics 7 306-10

[2] Sturmberg B C P, Dossou K B, Botten L C, Asatryan A A, Poulton C G, McPhedran R C and de Sterke C M 2014 Optimizing Photovoltaic Charge Generation of Nanowire Arrays: A Simple Semi-Analytic Approach ACS Photonics 1 683-9

[3] Kavanagh K L 2010 Misfit dislocations in nanowire heterostructures Semicond. Sci. Technol. 25024006

[4] Kubo S, Iwata S, Kurai S, Taguchi T, Kainosho K and Yokohata A 2004 Homoepitaxial growth of GaN layers by reactive molecular-beam epitaxy on bulk GaN single crystals prepared by pressure-controlled solution growth Jpn. J. Appl. Phys. 437454

[5] Nam O-H, Bremser M D, Zheleva T S and Davis R F 1997 Lateral epitaxy of low defect density GaN layers via organometallic vapor phase epitaxy Appl. Phys. Lett. $712638-40$

[6] Yonenaga I, Ohno Y, Taishi T, Tokumoto Y, Makino H, Yao T, Kamimura Y and Edagawa K 2011 Optical properties of fresh dislocations in GaN J. Cryst. Growth 318 415-7

[7] Albert S, Bengoechea-Encabo A, Zuniga-Perez J, de Mierry P, Val P, Sanchez-Garcia M A and Calleja E 2014 Selective area growth of GaN nanostructures: A key to produce high quality (11?20) a-plane pseudosubstrates Appl. Phys. Lett. 105091902

[8] Koester R, Hwang J-S, Salomon D, Chen X, Bougerol C, Barnes J-P, Dang D L S, Rigutti L, de Luna Bugallo A, Jacopin G, Tchernycheva M, Durand C and Eymery J 2011 M-Plane Core-Shell InGaN/GaN Multiple-Quantum-Wells on GaN Wires for Electroluminescent Devices Nano Lett. 11 4839-45

[9] Li Y, Xiang J, Qian F, Gradec?ak S, Wu Y, Yan H, Blom D A and Lieber C M 2006 Dopant-Free GaN/AlN/AlGaN Radial Nanowire Heterostructures as High Electron Mobility Transistors Nano Lett. 6 $1468-73$

[10] Lorenzo Rigutti, Gwénolé Jacopin, Andres De Luna Bugallo, Maria Tchernycheva, Elias Warde, François H Julien, Rudeesun Songmuang, Elisabeth Galopin, Ludovic Largeau and and Jean-Christophe Harmand 2010 Investigation of the electronic transport in GaN nanowires containing GaN/AlN quantum discs Nanotechnology 21425206

[11] E. Monroy, F. Calle, J.L. Pau, E. Muñoz, F. Omnès,B. Beaumont, P. Gibart 2001 AlGaN-based UV photodetectors J. Cryst. Growth $230537-43$

[12] E Monroy, F Omnès and F Calle 2003 Wide-bandgap semiconductor ultraviolet photodetectors Semicond. Sci. Technol. 18 R33-51

[13] Chen R S, Tsai H Y, Huang Y S, Chen Y T, Chen L C and Chen K H 2012 Photoconduction efficiencies and dynamics in $\mathrm{GaN}$ nanowires grown by chemical vapor deposition and molecular beam epitaxy: A comparison study Appl. Phys. Lett. 101113109

[14] Gonzàlez-Posada F, Songmuang R, Den Hertog M and Monroy E 2012 Room-Temperature Photodetection Dynamics of Single GaN Nanowires Nano Lett. 12 172-6 
[15] J. Lähnemann, M. Den Hertog,P. Hille, M. De la Mata, T. Fournier, J. Schörmann, J. Arbiol, M. Eickhoff, E. Monroy, 2016 UV Photosensing Characteristics of Nanowire-Based GaN/AlN Superlattices Nano Lett. $163260-7$

[16] L. Rigutti, M. Tchernycheva, A. De Luna Bugallo, G. Jacopin, F. H. Julien,L. F. Zagonel, K. March, O. Stephan, M. Kociak and R. Songmuang 2010 Ultraviolet Photodetector Based on GaN/AlN Quantum Disks in a Single Nanowire Nano Lett. $102939-43$

[17] Tchernycheva M, Neplokh V, Zhang H, Lavenus P, Rigutti L, Bayle F, Julien F H, Babichev A, Jacopin G, Largeau L, Ciechonski R, Vescovi G and Kryliouk O 2015 Core-shell InGaN/GaN nanowire light emitting diodes analyzed by electron beam induced current microscopy and cathodoluminescence mapping Nanoscale 7 11692-701

[18] Piazza V, Vettori M, Ali A, Lavenus P, Bayle F, Chauvin N, Julien F H, Regreny P, Patriarche G, Fave A, Gendry M and Tchernycheva M 2018 Nanoscale investigation of radial p-n junction in self-catalyzed GaAs NWs grown on $\mathrm{Si}(111)$ Nanoscale

[19] Donatini F, de Luna Bugallo A, Tchoulfian P, Chicot G, Sartel C, Sallet V and Pernot J 2016 Comparison of Three E-Beam Techniques for Electric Field Imaging and Carrier Diffusion Length Measurement on the Same Nanowires Nano Lett. 16 2938-44

[20] Spies M, den Hertog M I, Hille P, Schörmann J, Polaczyński J, Gayral B, Eickhoff M, Monroy E and Lähnemann J 2017 Bias-Controlled Spectral Response in GaN/AlN Single-Nanowire Ultraviolet Photodetectors Nano Lett. 17 4231-9

[21] L. Largeau, E. Galopin, N. Gogneau, L. Travers, F. Glas, J-C. Harmand 2012 N-Polar GaN Nanowires Seeded by Al Droplets on Si(111) Cryst Growth Des 2724

[22] L. Largeau, D.L. Dheeraj, M. Tchernycheva, G.E. Cirlin, J-C. Harmand 2008 Facet and in-plane crystallographic orientations of GaN nanowires grown on Si (111) Nanotechnology 19155704

[23] Laneuville V, Demangeot F, Péchou R, Salles P, Ponchet A, Jacopin G, Rigutti L, de Luna Bugallo A, Tchernycheva M, Julien F H, March K, Zagonel L F and Songmuang R 2011 Double strain state in a single GaN/AlN nanowire: Probing the core-shell effect by ultraviolet resonant Raman scattering Phys. Rev. B 83

[24] Galopin E, Largeau L, Patriarche G, Travers L, Glas F and Harmand J C 2011 Morphology of self-catalyzed $\mathrm{GaN}$ nanowires and chronology of their formation by molecular beam epitaxy Nanotechnology 22245606

[25] Calarco R, Marso M, Richter T, Aykanat A I, Meijers R, v.d. Hart A, Stoica T and Lüth H 2005 Sizedependent Photoconductivity in MBE-Grown GaN-Nanowires Nano Lett. 5 981-4

[26] Davydov A V, Motayed A, Boettinger W J, Gates R S, Xue Q Z, Lee H C and Yoo Y K 2005 Combinatorial optimization of Ti/Al/Ti/Au ohmic contacts to n-GaN Phys. Status Solidi C 2 2551-4

[27] Leamy H J 1982 Charge collection scanning electron microscopy J. Appl. Phys. 53 R51-80

[28] Yakimov E B, Borisov S S and Zaitsev S I 2007 EBIC measurements of small diffusion length in semiconductor structures Phys. Tech. Poluprovodiekov 41

[29] Togonal A S, Foldyna M, Chen W, Wang J X, Neplokh V, Tchernycheva M, Nassar J, Roca i Cabarrocas P and Rusli 2015 Core-Shell Heterojunction Solar Cells Based on Disordered Silicon Nanowire Arrays $J$. Phys. Chem. C $1202962-72$ 
[30] Baird L, Ong C P, Cole R A, Haegel N M, Talin A A, Li Q and Wang G T 2011 Transport imaging for contact-free measurements of minority carrier diffusion in $\mathrm{GaN}, \mathrm{GaN} / \mathrm{AlGaN}$, and GaN/InGaN core-shell nanowires Appl. Phys. Lett. 98132104

[31] Schuck P J, Mason M D, Grober R D, Ambacher O, Lima A P, Miskys C, Dimitrov R and Stutzmann M 2001 Spatially resolved photoluminescence of inversion domain boundaries in GaN-based lateral polarity heterostructures Appl. Phys. Lett. 79 952-4

[32] Liu R, Bell A, Ponce F A, Chen C Q, Yang J W and Khan M A 2005 Luminescence from stacking faults in gallium nitride Appl. Phys. Lett. 86021908

[33] Rigutti L, Jacopin G, Largeau L, Galopin E, De Luna Bugallo A, Julien F H, Harmand J-C, Glas F and Tchernycheva M 2011 Correlation of optical and structural properties of GaN/AlN core-shell nanowires Phys. Rev. B $\mathbf{8 3}$

[34] L F Zagonel, L Rigutti, M Tchernycheva, G Jacopin, R Songmuang and M Kociak 2012 Visualizing highly localized luminescence in GaN/AlN heterostructures in nanowires Nanotechnology 23 455205-12

[35] Luiz Fernando Zagonel, Stefano Mazzucco, Marcel Tencé, Katia March, Romain Bernard,Benoît Laslier, Gwénolé Jacopin, Maria Tchernycheva, Lorenzo Rigutti, Francois H. Julien, Rudeesun Songmuang, and Mathieu Kociak 2011 Nanometer Scale Spectral Imaging of Quantum Emitters in Nanowires and Its Correlation to Their Atomically Resolved Structure Nano Lett. 11 568-73

[36] Chen H-L, Himwas C, Scaccabarozzi A, Rale P, Oehler F, Lemaître A, Lombez L, Guillemoles J-F, Tchernycheva M, Harmand J-C, Cattoni A and Collin S 2017 Determination of n-Type Doping Level in Single GaAs Nanowires by Cathodoluminescence Nano Lett. 17 6667-75

[37] Himwas C, Collin S, Rale P, Chauvin N, Patriarche G, Oheler F, Julien F H, Travers L, Harmand J-C and Tchernycheva M 2017 In situ passivation of GaAsP NWs Nanotechnology

[38] Himwas C, Den Hertog M, Si Dang L, Monroy E and Songmuang R 2014 Alloy inhomogeneity and carrier localization in AlGaN sections and AlGaN/AlN Nanodisk in NWs with 240-350 nm emission Appl. Phys. Lett. 105

[39] L. F. Zagonel, L. H. G. Tizei, G. Z. Vitiello, G. Jacopin, L. Rigutti, M. Tchernycheva,F. H. Julien, R. Songmuang, T. Ostasevicius, F. de la Peña, C. Ducati, P. A Midgley, M. Kociak 2016 Nanometre scale monitoring of the quantum confined stark effect and emission efficiency droop in multiple GaN/AlN quantum disks in nanowires Phys. Rev. B 93205410

[40] Adelmann C, Sarigiannidou E, Jalabert D, Hori Y, Rouvière J-L, Daudin B, Fanget S, Bru-Chevallier C, Shibata T and Tanaka M 2003 Growth and optical properties of GaN/AlN quantum wells Appl. Phys. Lett. 82 4154-6

[41] Renard J, Songmuang R, Tourbot G, Bougerol C, Daudin B and Gayral B 2009 Evidence for quantumconfined Stark effect in GaN/AlN quantum dots in nanowires Phys. Rev. B $\mathbf{8 0}$ 